\title{
Model Validation: Robot Behavior in People Guidance Mission using DTM model and Estimation of Human Motion Behavior.
}

\author{
Anaís Garrell and Alberto Sanfeliu \\ Institut de Robòtica i Informàtica Industrial (CSIC-UPC) \\ 08028 Barcelona, Spain
}

\begin{abstract}
This paper describes the validation process of a simulation model that have been used to explore the new possibilities of interaction when humans are guided by teams of robots that work cooperatively in urban areas. The set of experiments, which have been recorded as video sequences, show a group of people being guided by a team of three people (who play the role of the guide robots). The model used in the simulation process is called Discrete Time Motion model (DTM) described in [7], where the environment is modeled using a set of potential fields, and people's motion is represented through tension functions. The video sequences were recorded in an urban space of $10.000 \mathrm{~m}^{2}$ denominated Barcelona Robot Lab, where people move in the urban space following diverse trajectories. The motion (pose and velocity) of people and robots extracted from the video sequences were compared against the predictions of the DTM model. Finally, we checked the proper functioning of the model by studying the position error differences of the recorded and simulated sequences.
\end{abstract}

\section{INTRODUCTION}

Nowadays, research community have increased its interest in robots performing social tasks, consequently the needed of studying new abilities for human-robot interaction and task cooperation have become important issues. One application where these abilities are shown is in guiding a group of people using one or several robots [4]. Some experiments of guiding people with one robot have been already performed, but when is a group of people guided by a team of robots, the experiments are not performed easily due to the limitations of the present technology (robots do not move or change their orientation fast enough). In these situations is better to simulate the experiments and then validate the results using sequence of real guided missions [2].

In this work, we present the validation of a model described previously in [7], denominated "Discrete-TimeMotion" model (DTM). The DTM model is a new approach for guiding people in open areas of urban settings using multiple robots acting in a cooperative way. One of the robots is the leader, and acts as a human tour-guide. It is placed at the front of the formation and its role is to estimate the trajectory of both people and the rest of robots. The other robots, called shepherd, are responsible for guiding people,

This research was conducted at the Institut de Robòtica i Informàtica Industrial (CSIC-UPC). It was partially supported by CICYT projects DPI2007-61452 and Ingenio Consolider CSD2007-018, by CSIC project 200850 I055 and by IST-045062 of European Community. The first author acknowledges Spanish FPU grant ref. AP2006-00825.

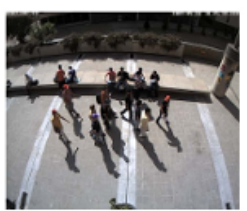

(a)

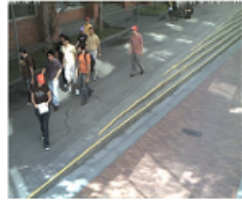

(b)

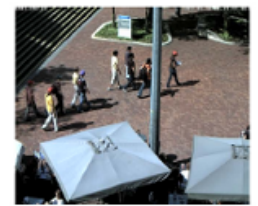

(c)

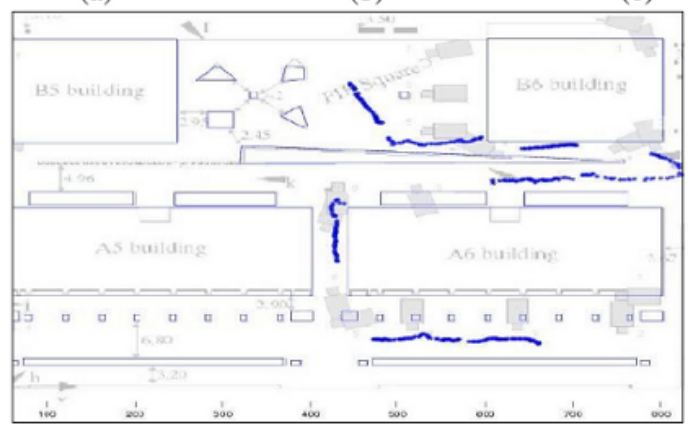

(d)

Fig. 1. (a), (b) and (c) show different captions of the guided group by the camera network. (d) shows an aerial view of the experimental site, Barcelona Robot Lab, including the distribution of camera network, and the trajectory of the group in one of the experiments.

preventing any person leaving the group, and following the path given by the leader. The DTM model represents robots, people and obstacles by means of potential functions, and it computes the estimations of people's and robots positions. The model uses a particle filter [1] for positions estimation, and it considers Helbing equations [8], [9] for the simulation of human reactions in front of different stimulus that come from the robots and the constraints of the environment.

The works presented in the literature show their contributions for groups of robots that interact with people using simulations. For instance, [6] performs a qualitative analysis of the movements of different entities -such as humans or animals- and build an architecture of three robots to guide them. However, realistic situations, such as the existence of obstacles or dealing with people leaving the group are not considered, and their models were not validated. In [13] several types of robot formations and different strategies for approaching the robots to people are considered. However. all these issues and the general movements of the robots are ruled by a large number of heuristics which makes the system impractical. Furthermore, in order to achieve the 
desired guiding results, complex robot motions (for example the acceleration must be to high) are required.

In contrast to the previous approaches, the "DiscreteTime-Motion" model proposed previously in [7], offers a framework to tackle more realistic situations and without the need of using such a large number of heuristics. Moreover, this model can tackle realistic situations such as avoid people escaping from the group.

For the validation of this research, we used the network of cameras installed in the Barcelona Robot Lab, in the Universitat Politècnica de Catalunya (UPC), an area of 10.000 $m^{2}$ in the north Campus. We performed a set of experiments where a group of people were guided by three people that play the role of the guide robots. They have performed 9 experiments following the same path, but there were some differences in each experiment. The group made the same trajectory nine times, and in each one the group presented different behaviors, for instance, a person escaped from the group and must be regrouped, or some people escaped in different directions. These human behaviors were simulated and we studied how the behavior of the robots must be for achieving their objective, this is, lead the group towards the goal. In the present paper, we validate the DTM model by analyzing the position and velocity error differences of people and robots.

In the remainder of the paper we start by describing shortly the Discrete-Time-Motion model used in the simulations. Section III presents how the data was collected and how the validation process was done. Results and conclusions are presented in sections IV and V, respectively.

\section{REVIEW OF DISCRETE TIME MOTION MODEL}

In this section, we will present a short review of the Discrete Time Motion (DTM) model, which, on one hand, it models the representation of the whole environment, and, on the other hand, it estimates at each time instance the position and velocity of every human and robot, as well as to predict their future states. The DTM evaluates these data in discrete time instances, every $\mathrm{N}$ units of time (seconds or milliseconds).

The DTM model considers that one of the robots is the leader and act as a tour guide. It is allocated at the front of the formation and its mission is to assure that all the group arrives to the desired goal. The rest of robots, called shepherds, have to maintain the formation and assure that no individual escapes from the group. This model has two components: The Discrete Time component and the Discrete Motion component.

The Discrete Time component uses the potential fields for people, robots and obstacles representation (fixed or mobile). We call tensions to these potential fields applied to people, robots or obstacles. A fixed obstacle has a very high tension in a determined area which decreases with the distance to the center of the object (the tensions are computed based on the area defined by a security region surrounding each human, robot and obstacle). A person or a robot can move to a specific position if the tension in that place is very low or zero.

In order to decide the trajectories the robots will follow, we define a potential field over the working area, and we perform a path planning in it [11], [12]. Then, we define a set of attractive and repulsive forces. In particular, the goal the robots try to reach generate an attractive force pulling the robots towards it. On the other hand, the obstacles generate a repulsive potential pushing a given robot away. The rest of robots and people generate similar repulsive forces, although, with less intensity than the obstacle's forces.

We parameterized all these attractive and repulsive forces by Gaussian functions. For instance, the repulsive forces for people are:

$$
T_{p}\left(\mu_{p}, \Sigma_{p}\right)(x)=\frac{1}{\left|\Sigma_{p}\right|^{1 / 2}(2 \pi)^{n / 2}} e^{-\frac{1}{2}\left(x-\mu_{p}\right)^{T} \Sigma_{p}^{-1}\left(x-\mu_{p}\right)}
$$

where $\mu_{p}=\left(\mu_{p_{x}}, \mu_{p_{y}}\right)$ is the center of gravity of the person, and $\Sigma_{p}$ is a covariance matrix whose principal axes $\left(\sigma_{x}, \sigma_{y}\right)$ represent the size of an ellipse surrounding the person which is used as a security area. A similar expression defines the potential map associated for each robot. These repulsive forces may be interpreted as continuous probability functions over the entire space.

We can then define people and robots by the set $\left\{\left(\mu_{x}, \mu_{y}\right),\left(\sigma_{x}, \sigma_{y}\right), v, \theta, T\right\}$, where $v$ and $\theta$ are the velocity and orientation computed by the particle filter and $T$ is the associated tension. As we said, the variances $\left(\sigma_{x}, \sigma_{y}\right)$ represent the security area around each individual.

In the case of obstacles, we define their tension as a set of Gaussian functions placed at regular intervals around their boundaries. Let us denote by $X=\left\{\left(x_{1}, y_{1}\right), \ldots,\left(x_{n}, y_{n}\right)\right\}$ the set of points evenly spaced around the boundary. Then this boundary will be defined by: $\left\{\left(x_{i}, y_{i}\right),\left(\sigma_{x_{i}}, \sigma_{y_{i}}\right), T_{i}\right\}$ for $i=$ $1, \ldots, n$, where $T_{i}$ follows Eq. 1 .

After having defined the tensions for each of the components of the environment we are ready to define the potential field of all the environment space. This is easily computed as the intersection of all the Gaussian functions for a given variances.

The second component, the Discrete Motion Component, will decide the motion strategies to be followed by the robots in order to achieve their goals. Robot motion is computed using a simple path planning algorithm [10]. This path planning is only performed by the leader robot who transmits the computed path to other robots.

The second case is the study of shepherding algorithms [3], [5], which are inspired in the shepherd dogs. The shepherding task is performed by all the robots except by the leader.

One important situation we must carefully consider is the case when people escape from the group. We are not aware of any approach tackling this problem. The solution we adopt for this situation is to choose one of the robots -the one closer to the individual who left- and bring him back to the formation. For computing the trajectory that 


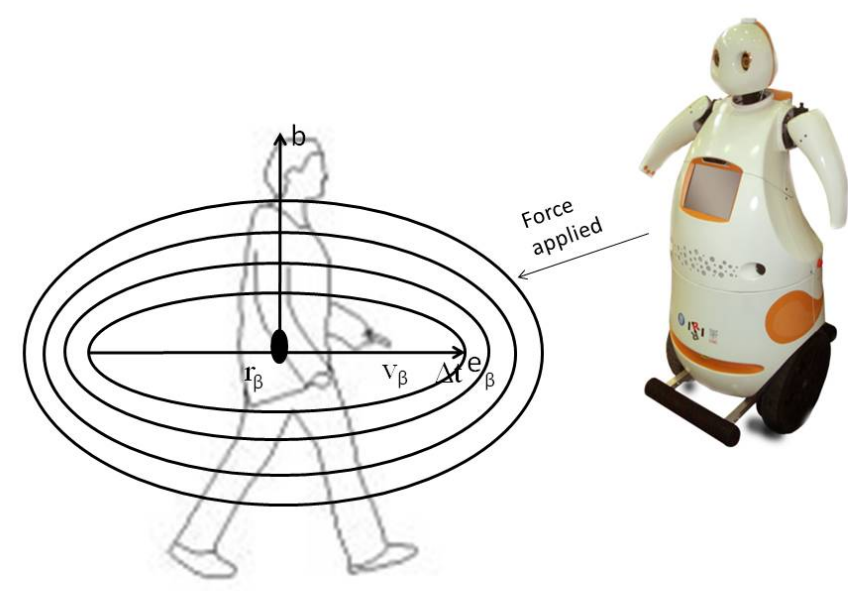

Fig. 2. Parameters of people's vital space.

will be considered for intercepting the person who escaped from the group, we first use a Particle Filter to estimate the position and velocity of the person and then we compute the interception point.

In order to model people's motion we use the concepts introduced by Helbing et al. [8], [9], which studies the dynamics of pedestrian crowds from the "social" point of view. More specifically, they describe the motion of pedestrians based on social forces which are the result of the internal motivations of the individuals to perform specific motions.

Firstly, the model considers that people want to reach a specific destination as comfortable as possible which may be formally represented as the shape of a polygon with edges $\vec{r}_{\alpha}^{1} \ldots \vec{r}_{\alpha}^{n}:=\vec{r}_{\alpha}^{0}$, where $\alpha$ refers to a given person and $\vec{r}_{\alpha}^{0}$ the destination he/she wants to reach.

Secondly, we have to consider the situation when the pedestrian motion is influenced by other pedestrians from the group. This situation responds to the fact that each individual tries to maintain an empty security volume surrounding him [16]. This is in fact a repulsive effect which we model through the following vectorial quantity:

$$
\vec{F}_{\alpha \beta}\left(\vec{r}_{\alpha \beta}\right)=-\nabla_{\vec{r}_{\alpha \beta}} V_{\alpha \beta}\left[b\left(\vec{r}_{\alpha \beta}\right)\right]
$$

where $V_{\alpha \beta}(b)$ is a repulsive potential which is assumed to be a monotonic decreasing function of $b$ with equipotential lines having an elliptical shape. $\vec{r}_{\alpha \beta}=\vec{r}_{\alpha}-\vec{r}_{\beta}$. The parameter $b$ denotes the semi-minor axis of the ellipse and is given by:

$$
b=\frac{\sqrt{\left(\left\|\vec{r}_{\alpha \beta}\right\|+\left\|\vec{r}_{\alpha \beta}-v_{\beta} \Delta t \vec{e}_{\beta}\right\|\right)^{2}-\left(v_{\beta} \Delta t\right)^{2}}}{2}
$$

where, $v_{\beta} \Delta t$ is an approximation to the step size of a pedestrian $\beta$.

Finally, we consider the repulsive effect produced by the distance that people try to keep away from the obstacles of the environment. The nature of this force is the same we just described between individuals, with the difference that the obstacles do not move. If we denote by $B$ the border of an obstacle the repulsive effect it creates will be described

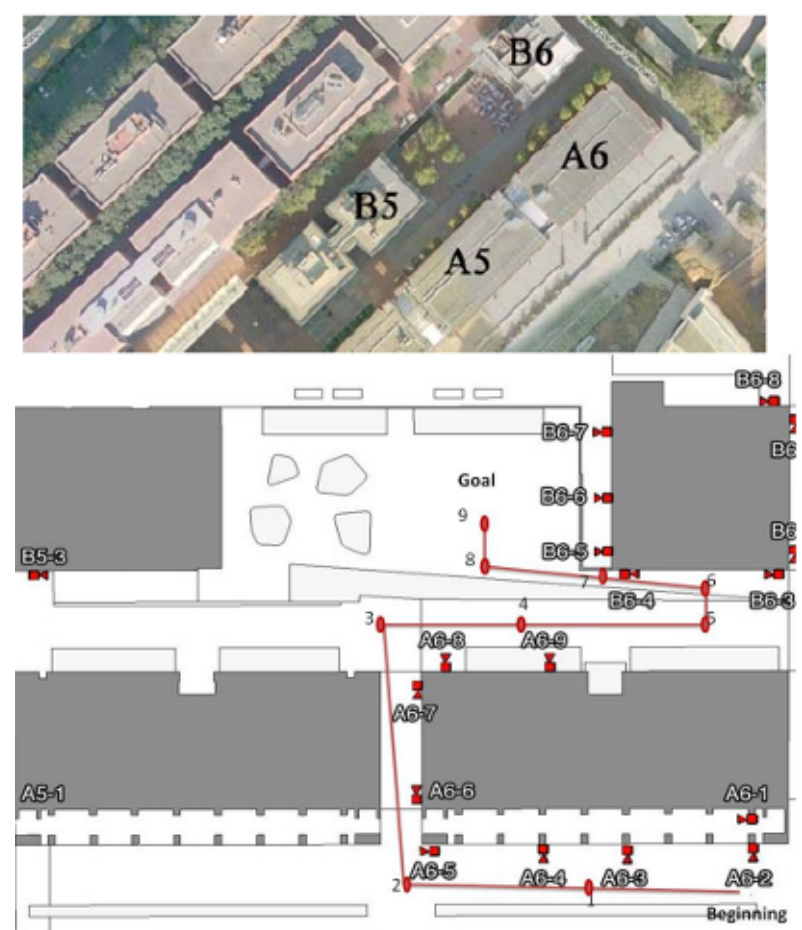

Fig. 3. An aerial view of our experimental site, the Barcelona Robot Lab (top), and the distribution of cameras in the network with the route that the group follows (bottom).

by:

$$
\vec{F}_{\alpha B}\left(\vec{r}_{\alpha B}\right)=-\nabla_{\vec{r}_{\alpha B}} U_{\alpha B}\left(\left\|\vec{r}_{\alpha B}\right\|\right)
$$

where $U_{\alpha B}\left(\left\|\vec{r}_{\alpha B}\right\|\right)$ is the repulsive and monotonically decreasing potential function, $\vec{r}_{\alpha B}=\vec{r}_{\alpha}-\vec{r}_{B}$, and $B$ denotes the position of the border $B$ that is closest to the pedestrian $\alpha$.

We have presented the Discrete Time Motion model which has been used for the development of simulations, and whose validation process is presented below.

\section{DATA COLLECTION AND VALIDATION PROCESS}

In this section, we explain the method for data collection, which is used for the validation and testing of the DTM model. Model validation is used for verifying that the model, within its domain of applicability, behaves consistently with the objectives. Model validation deals with building the right model. In model testing, the model is subjected to test data or tests cases to determine if it functions properly. Test failed implies the failure of the model, not the test. Some tests are devised to evaluate the behavioral accuracy (i.e., validity) of the model, and some tests are intended to judge the accuracy of model transformation from one form into another (verification). In the present work we assess how our model behaves in a set of real situations. But, before explaining the validation and testing process we proceed to the description of data collection.

For data collection we use the camera network mounted on the Barcelona Robot Lab, composed by 21 interconnected cameras, see Fig. 3, which took a set of video sequences of 

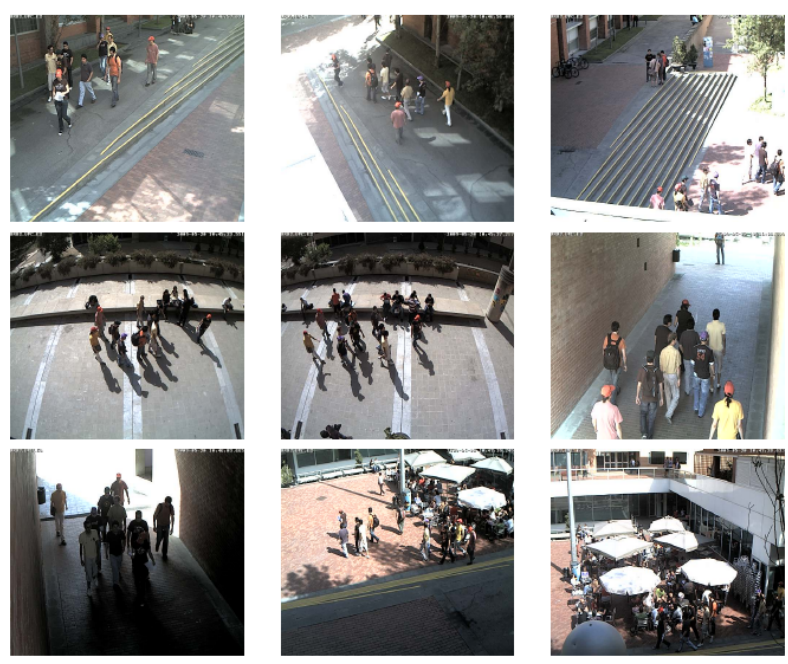

Fig. 4. Images of the set of cameras of the Barcelona Robot Lab. Different frames of the group of people being guided by the three members that play the role of guide robots.

the group of people being guided by three other people (who play the role of guide robots). The tour, depicted in Fig. 1, was performed nine times and in each one of the trials, some people of the group behaved in different ways. Below are some examples of the people and guides behavior:

- The group follows the instructions of the guides always.

- A person who is being led, goes away from the group and one of the guides has to look forward him in order to regroup him in the main group.

- Several people escape at the same time in opposite directions.

- The group stops and moves again.

- The guides are placed at specific locations to create a robot barrier, for example at corners to prevent people escaping.

Different routes were done using this set of behaviors and they were used for validating the model simulations. The data collection process was the following: (1) the video sequences were registered; (2) each frame of the video sequences was rearranged in order to synchronize the complete sequence, complete path was registered by 15 cameras; (3) positions of people was annotated manually. More than 10,000 images were labeled. Furthermore, using [14] we transformed the image's coordinates to map's coordinates for each frame of the sequence, see Fig. 1. Finally, all the different paths were joined to get people's trajectories. As shown in Fig. 1, there are some areas where the information of people's trajectory is lacking, and in these areas we are not able to do any comparison.

For the model validation process, the trajectory of every person has to be taken into account in the complete path, as well as the trajectory of all the group. This trajectories are then compared against the estimation obtained by the simulations using the DTM model. The comparison is based on the quadratic error of the real (from the video sequence) and estimated (from the simulation) positions. Two cases were studied: the motion behavior of the shepherd and leader robots; and the motion behavior of the guided people.

For robots, several cases were analyzed. For the leader we examine the motion trajectory that it has followed for guiding the group and the velocity at each position. For the shepherd we compute the motion trajectory for following the group and the motion trajectory to look for a person that escapes from the formation and he has to be regrouped. For the guided people we compute the estimated trajectory using the particle filter against the observations obtained from the camera network.

All this comparison are done in the areas where the camera network have information of the position of people and robots. There are some areas where the cameras are not able to see people's trajectories due to environment constraints. In the areas where there are not real data, we only can estimate their positions, and the error will be computed at the next position obtained from the camera network.

Here, we present the formulation of the quadratic error that will be used to evaluate the usefulness and truthfulness of the simulations using the DTM. The quadratic error is computed for each motion instant $k$, where $y_{k}$ is the data obtained by camera network, for instance, velocity or position of a person or robot, and $\hat{y}_{k}$ is the value given by the DTM model. The quadratic error at time $k$ is given by:

$$
\text { error }_{k}=\sqrt{\left(y_{k}-\hat{y}_{k}\right)^{2}}
$$

In next section we will present the obtained results in simulations and the study of the quadratic error between the simulated and observed data.

\section{EXPERIMENTS AND RESULTS}

The current work is done within the framework of the European Project URUS [15], and the scenario where the experiments will be performed corresponds to an urban area of about $10.000 \mathrm{~m}^{2}$ within the North Campus of the Universitat Politècnica de Catalunya (UPC). The area contains different obstacles, such as buildings, benches and trash cans.

As we have explained above, we have created a database of people and robot positions and velocities taken from a the set of sequences of images registered by the camera network at the Barcelona Robot Lab. A group of 7 people were led by three people playing the role of guide robots, where one performed the role of Leader, as a tour guide, and other two performed the functions of assistants. In addition, we have obtained different human behaviors in the set of scenes, for instance, a person escaping from the group or a group of people not following the leader.

The results correspond to the comparison between the simulated experiments and the real observation in the Barcelona Robot Lab. We assumed a team of three robots, which will move according to the motion model computed in Section II.

The experiment has three distinctive situations: (1) an area where the group of people is moving in an open area with no 
obstacle, and people is able to move freely, Fig. 5; (2) people has to do a curve of 180, Fig. 6; and, finally, (3) in last scene the group is moving through a corridor, here the environment is bounded and people motion is more controlled, Fig. 7 .

In the image sequences Fig. 5-9 the leader robot, the shepherd robots and the people motion behavior are shown in three different environments. The left hand images represent the path done by the group of people or robots. The right hand images plot the evolution of the quadratic error (in $\mathrm{dm})$ through time. The error is computed at each frame, however the position of people and robots is corrected every ten seconds. Fig. 5-7 shows the trajectories of two different people and their position estimations using DTM model. Note that the error of the trajectory is high in Fig.5 because people start moving. In the rest of the figures, the error varies due to the uncertain movement of the person. Although that, the DTM model shows a very accurate in the estimation.

Fig. 8 shows the comparisons of the leader behavior. The leader robot in the DTM model computes the trajectory using a path planning, and the rest of the group must follow it. As we can see, the planned trajectory is linear in the straight paths, however the trajectory followed by the person whose role is the leader robot follows a curvilinear trajectory, which indeed is a typical human behavior. However, both behaviors are quite similar.

Last but not least, Fig.9 shows shepherd robots behavior depending on people trajectories. Fig.9 shows different situations of the shepherd robots: (a) shows the shepherd robots (red color) following the group (green color); (c) shows one shepherd robot (red color upper trajectory) that follows the group, and the second one is following one person that goes away from the group and brings him to the group; and (e) is similar to Fig. 9(a).

In conclusion, the study has shown that simulations of the DTM model are very close to the real motion of people who are being guided and very similar to people that play the role of robots. The average error is less than 1 meter.

\section{CONCLUSIONS AND FUTURE WORKS}

We have presented the validation of the DTM model to guide people in urban areas with a set of mobile robots working in a cooperative manner. In contrast to existing approaches, our method can tackle more realistic situations, such as dealing with large environments with obstacles, or regrouping people who left the group.

We have analyzed different situations: guiding in open areas, in corridors or in curves. In all these experiments we have compared our simulations with data obtained by camera network, and the results show that the error is very low (in average one meter) between the simulation and the real data. In the case of escaping people, the analysis shows that the shepherd robots follow a similar trajectory of a person playing the role of the robot.

We are currently working in improving the strategies with several robots. Firslty, we are studying people behavior when are trying to escape from de formation. Furthermore, we plan to study which is the optimal number of robots
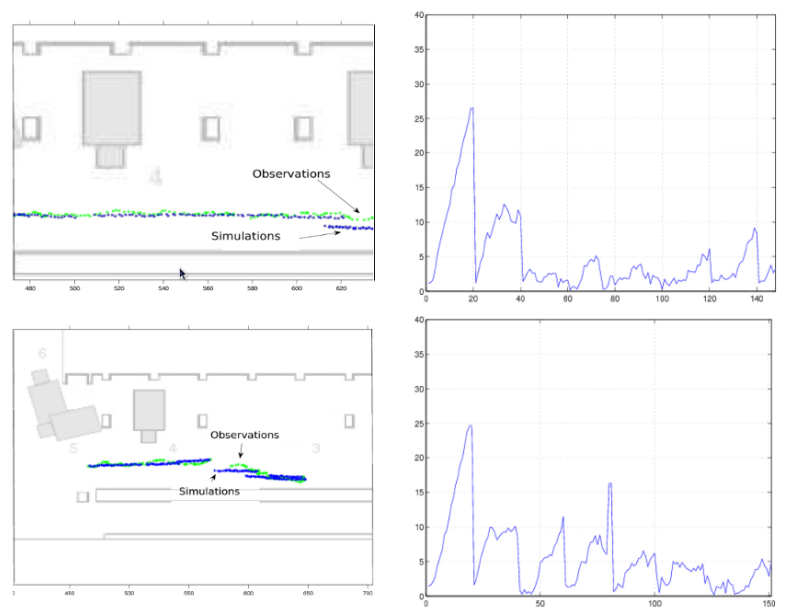

Fig. 5. On left hand, two individual trajectories in an open area are represented along with its estimations, on right hand, error evolution is shown, we can observe that the error decreases in time, at the beginning we can see that error is greater because of acceleration.
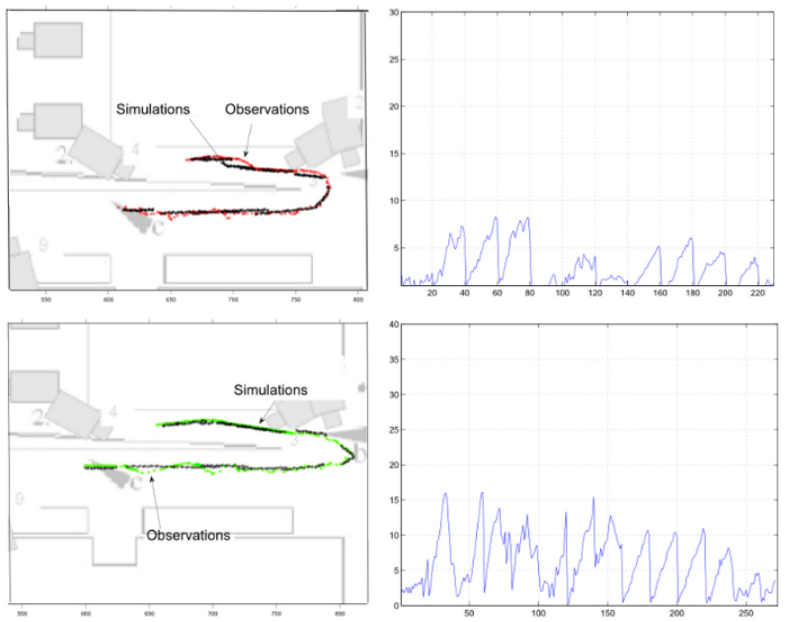

Fig. 6. On left hand, two individual trajectories in close curve are represented along with its estimations, on right hand, error evolution is shown.

depending on the number of people and the configuration of the environment. We also plan to add the path planning capabilities to the shepherd robots, and not only to the leader. Finally, we are improving the robot trajectories to fit better with the human trajectories.

\section{REFERENCES}

[1] M.S. Arulampalam, S. Maskell, N. Gordon, T. Clapp, D. Sci, T. Organ, and S.A. Adelaide. A tutorial on particle filters for online nonlinear/non-gaussianbayesian tracking. Signal Processing, IEEE Transactions on, 50(2):174-188, 2002.

[2] O. Balci. Verification validation and accreditation of simulation models. In Proceedings of the 29th conference on Winter simulation, pages 135-141. IEEE Computer Society Washington, DC, USA, 1997.

[3] C. Breazeal, A. Brooks, J. Gray, G. Hoffman, C. Kidd, H. Lee, J. Lieberman, A. Lockerd, and D. Mulanda. Humanoid robots as cooperative partners for people. Int.Journal of Humanoid Robots, 1(2), 2004. 

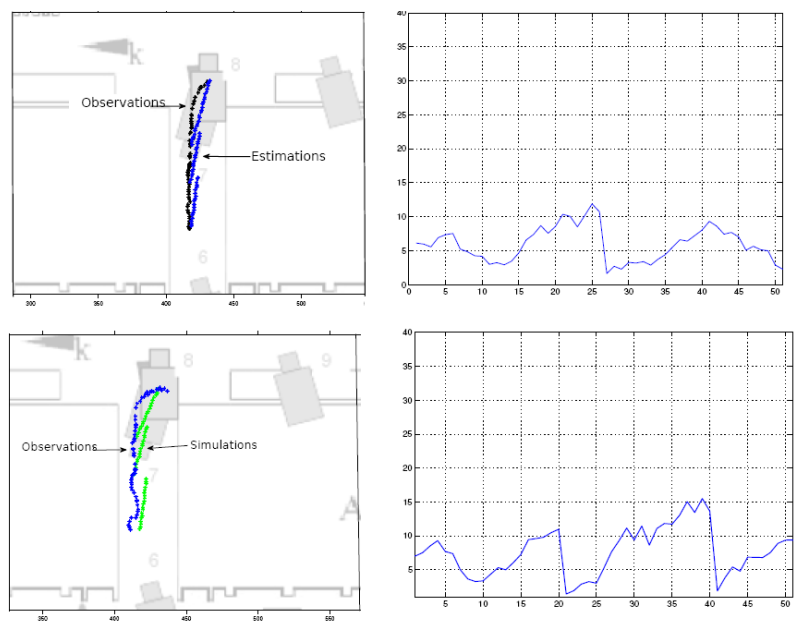

Fig. 7. On left hand, two individual trajectories in a corridor are represented along with its estimations, on right hand, error evolution is shown.

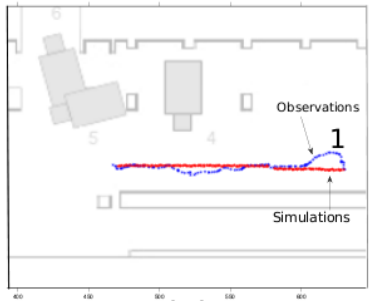

(a)

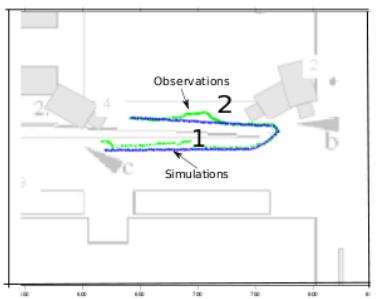

(c)

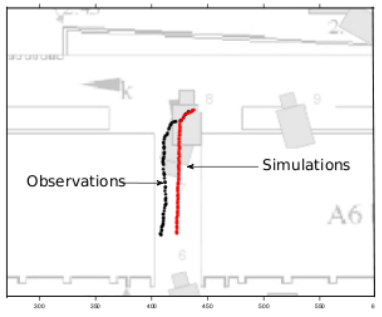

(e)

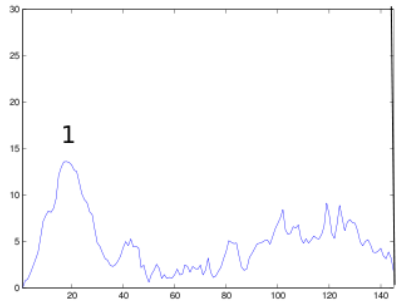

(b)

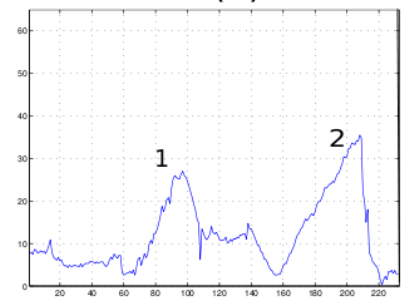

(d)

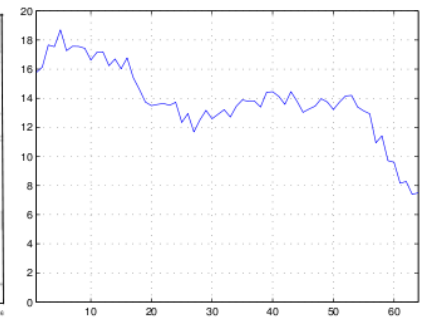

(f)
Fig. 8. Leader Robot behavior in three different scenes are shown, on left hand the comparison error between the simulated behavior and the observation data are represented. In (a) and (b) it must be considered that point 1 shows a great error due to the observation trajectory performs a curvilinear path while the simulated trajectory performs a curvilinear one, both trajectories are correct. IN (c) and (d) occurs a similiar behavior, at points 1 and 2 different path produce a higher error, again, both trajectories can be considered right.

[4] W. Burgard, A. B. Cremers, D. Fox, D. Hahnel, G. Lakemeyer, D. Schulz, W. Steiner, and S. Thrun. The interactive museum tourguide robot. In Proc.of the Fifteenth National Conference on Artificial Intelligence, Madison, Wi, 1998.

[5] L. Chaimowicz and V. Kumar. Aerial shepherds: Coordination among uavs and swarms of robots. Proceedings of the 7th International Symposium on Distributed Autonomous Robotic Systems, 2004.

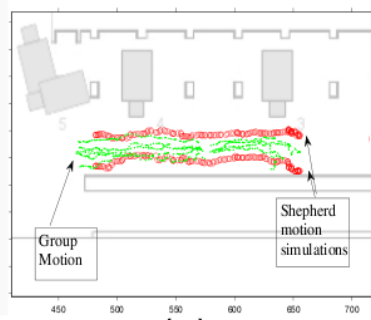

(a)

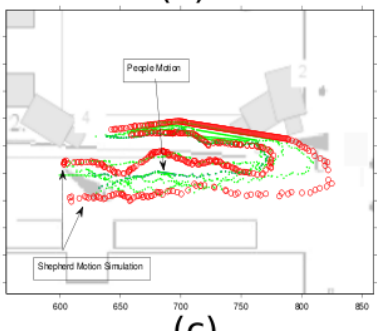

(c)

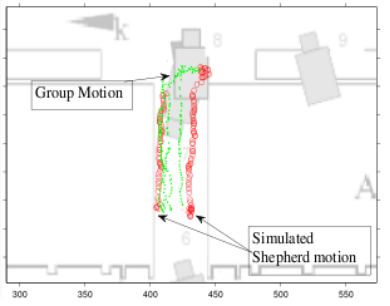

(e)

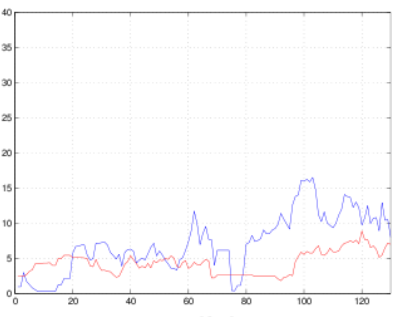

(b)

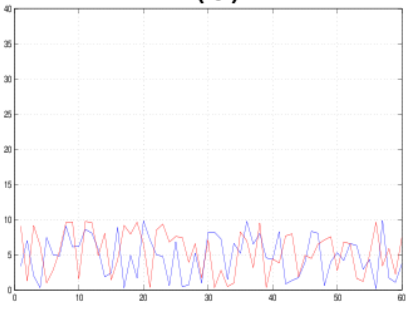

(d)

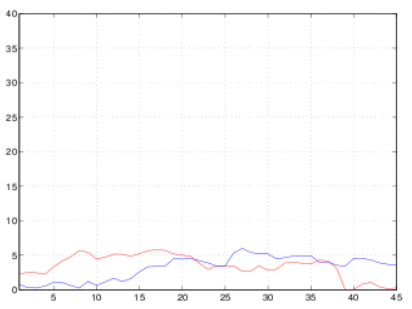

(f)
Fig. 9. Shepherd Robot behaviors and group motion in three different scenes are shown, on left hand the trajectories of shepherd robots and people who are guided are presented, on the right hand comparison error between the simulated behavior and the observation data are represented.

[6] Martinez Garcia E.A., Akihisa O., and S. Yuta. Crowding and guiding groups of humans by teams of mobile robots. Advanced Robotics and its Social Impacts, 2005.IEEE Workshop on, pages 91-96, 2005.

[7] A. Garrell, A.Sanfeliu, and F.Moreno-Noguer. Discrete time motion model for guiding people in urban areas using multiple robots. In IEEE/RSJ International Conference on Intelligent Robots and Systems., 2009.

[8] D. Helbing and P. Molnar. Social force model for pedestrian dynamics. Physical Review E 51, pages 4282-4286, 1995.

[9] D. Helbing and P. Molnar. Self-organization phenomena in pedestrian crowds, in self-organization of complex structures: From individual to collective dynamics. F. Schweitzer (Ed.) (Gordon and Breach, London), pages 569-577, 1997.

[10] C. Hofner and G. Schmidt. Path planning and guidance techniques for an autonomous mobilecleaning robot. Proc. Int. Conf. Intelligent Robots and Systems (IROS), 1, 1994.

[11] Y. K. Hwang and N. Ahuja. A potential field approach to path planning. IEEE Transactions on Robotics and Automation 8(1), 1992.

[12] S. M. LaValle. Rapidly-exploring random trees: A new tool for path planning. Computer Science Dept, Iowa State University, Tech.Rep.TR, pages $98-11,1998$.

[13] J.M. Lien, S. Rodriguez, J.P. Malric, and N.M. Amato. Shepherding behaviors with multiple shepherds. Proc. IEEE Int. Conf. Robot. Autom. (ICRA), 2005.

[14] A.A. Ortega, B. Dias, E.H. Teniente, A.J. Bernardino Malheiro, J. Gaspar, and J. Andrade-Cetto. Calibrating an outdoor distributed camera network using laser range finder data. IEEE/RSJ International Conference on Intelligent Robots and Systems, Saint Louis, pages 303308, 2009.

[15] A. Sanfeliu and J. Andrade-Cetto. Ubiquitous networking robotics in urban settings workshop on network robot systems. Proc. Int. Conf Intelligent Robots and Systems (IROS), 2006.

[16] S. Worchel, J. Cooper, G. R. Goethals, and J. M. Olson. Social psychology. Thomson Learning Ibero, 2002. 